\title{
Resonantly damped fast MHD kink modes in longitudinally stratified tubes with thick non-uniform transitional layers
}

\author{
I. Arregui, T. Van Doorsselaere, J. Andries ${ }^{\star}$, M. Goossens, and D. Kimpe
}

\author{
Centrum voor Plasma-Astrofysica, K.U. Leuven, Celestijnenlaan 200B, 3001 Heverlee, Belgium \\ e-mail: [Inigo.Arregui ; tomvd; Jesse.Andries; Marcel.Goossens; Dries.Kimpe]@wis.kuleuven. be
}

Received 10 March 2005 / Accepted 23 June 2005

\begin{abstract}
Resonantly damped fast kink quasi-modes are computed in fully resistive magnetohydrodynamics (MHD) for twodimensional equilibrium models. The equilibrium model is a straight cylindrically symmetric flux tube with a plasma density that is non-uniform both across and along the loop. The non-uniform layer across the loop is not restricted to be thin, but its thickness can reach values up to the loop diameter. Our results indicate that the period and damping of coronal loop oscillations mainly depend on the density contrast and the inhomogeneity length-scale and are independent of the details of longitudinal stratification, depending on the weighted mean density, weighted with the wave energy. For fully non-uniform loops, quasimodes can interact with resistive Alfvén eigenmodes leading to avoided crossings and gaps in the complex frequency plane. The present study extends previous studies on coronal loop oscillations in one-dimensional equilibrium models with thick boundary layers and in equilibria with longitudinally stratified loops under the thin boundary approximation, and allow for a better comparison between observations and theory raising the prospect of coronal seismology using the time damping of coronal loop oscillations.
\end{abstract}

Key words. magnetohydrodynamics (MHD) - methods: numerical - Sun: oscillations - Sun: magnetic fields

\section{Introduction}

Transverse coronal loop oscillations triggered by explosive events, such as flares or filament eruptions, were first observed by the EUV telescope on board the TRACE spacecraft (Aschwanden et al. 1999; Nakariakov et al. 1999). Since then, several similar events have been reported and studied (Aschwanden et al. 2002; Schrijver et al. 2002). These oscillations have been interpreted as MHD fast kink-modes of a cylindrical coronal flux tube by Nakariakov et al. (1999) and Nakariakov \& Ofman (2001). This interpretation in terms of discrete modes of oscillation naturally leads to the prospect of a proper development of coronal seismology, i.e. the determination of unknown physical properties of the coronal plasma by comparison of observed and predicted properties of waves and oscillations. Coronal seismology was first suggested by Uchida (1970) and Roberts et al. (1984) and has already been used for the determination of the magnetic field and transport coefficients in the corona (Nakariakov et al. 1999; Nakariakov \& Ofman 2001).

In most of the observed events of spatial transverse coronal loop oscillations the oscillatory amplitude has been found to be strongly damped, typically having an exponential decay time of a few oscillatory periods. This observational fact might have some important physical consequences, both for

* Postdoctoral Fellow of the National Fund for Scientific Research - Flanders (Belgium) (F.W.O. - Vlaanderen). coronal seismology as for wave based coronal heating theories, once we have identified the correct damping mechanism(s). However, the cause of the rapid damping is still a matter of considerable debate. Currently, several mechanisms are under study: non-ideal effects such as viscous and ohmic damping, optically thin radiation and thermal conduction; lateral wave leakage due to the curvature of the loops (Roberts 2000); mechanisms based on the topology of magnetic field lines, such as footpoint motions near separatrices (Schrijver \& Brown 2000); footpoint leakage through the chromospheric density gradient (De Pontieu et al. 2001; Ofman 2002); phase mixing of Alfvén waves (Heyvaerts \& Priest 1983; Nakariakov et al. 1999; Ofman \& Aschwanden 2002); and resonant absorption of waves (Hollweg \& Yang 1988; Goossens et al. 2002; Ruderman \& Roberts 2002; Aschwanden et al. 2003). Since first suggested by Ionson (1978) as a non-thermal coronal heating mechanism, resonant absorption of waves has extensively been studied in both analytical and numerical studies. For instance, Davila (1987) provided an improved method for calculating the resonant absorption heating rate based on a two-scale description of the wave motions. The dependence of the heating rate on resistive dissipation was studied by Steinolfson \& Davila (1993), who also stressed the importance of the global mode to carry out energy across magnetic surfaces, whilst the effects of viscous dissipation have been studied in detail by Ofman et al. (1994). Whether the derived decay rate gives a mode conversion rate or a heating rate has been the subject 
of considerable controversy, see for example Lee \& Roberts (1986) and references therein.

This paper concentrates on the ideal damping of the global kink oscillations. In this context, the rapid decay of oscillations in coronal loops was predicted well before they were observed (see Ionson 1978; Lee \& Roberts 1986; Grossmann \& Smith 1988; Hollweg \& Yang 1988, for example). For instance, Hollweg \& Yang (1988) estimated that these oscillations would be damped very effectively with an $e$-folding time of only two wave periods. As the damping rate of quasi-mode kink oscillations is independent of dissipation in the limit of vanishing dissipation (Poedts \& Kerner 1991), this mechanism is fully consistent with the classical estimates of very large coronal Reynolds numbers of the order of $10^{14}$ (Priest 1982). Observational tests with TRACE data, by Ofman \& Aschwanden (2002), revealed that the scaling law of the damping time as a function of other physical parameters, such as the loop length and the oscillation period, seem to favour phase mixing. However, as pointed out by Goossens et al. (2002), damping of quasi-mode kink oscillations gives a consistent explanation of the rapid decay of the observed loop oscillations in the sense that by assuming reasonable values of the unknown parameters (such as density contrast and inhomogeneity scale length) appropriate damping times can be obtained. However, the length-scales of the inhomogeneity deduced by Goossens et al. (2002), are in between 0.15 and 0.5 , and hence are not entirely consistent with the assumption of a thin transitional layer used to obtain them, pointing to the need to relax this assumption.

A computation of damped quasi-modes in fully nonuniform 1D equilibrium models was carried out by Van Doorsselaere et al. (2004, hereafter VD04), who computed the frequency and damping rate of quasi-modes in fully non-uniform equilibrium states. By solving the linearised resistive MHD equations, VD04 found that for low density contrasts and large inhomogeneity length-scales, as observed in the oscillating coronal loops (Aschwanden et al. 2003), the numerically computed damping rates can deviate by up to $25 \%$ from the approximate results predicted by the formula obtained under the "thin" boundary approximation. The results obtained by VD04 have been used for the first full scale observational test of resonant absorption. Aschwanden et al. (2003) find that the density ratio predicted by the damping times obtained with resonant absorption is a factor of $\simeq 1.2-3.5$ higher than the density ratio estimated from the background fluxes. These parameters are only determined indirectly through the emission measure and are thus subject to considerable modelling. Also, the inferred extremely large inhomogeneity length scales result in small values of the density contrast through the resonant absorption model. Taking into account these observational and theoretical limitations resonant absorption predicts damping times that are commensurable with the observed ones.

More recently, resonantly damped kink quasi-modes have been studied for equilibrium models with a variation of the density in both the radial and axial directions by Andries et al. (2005b). The variation of density across the loop was limited to a thin transitional layer in order to focus on the effect of longitudinal stratification. An important result of this investigation

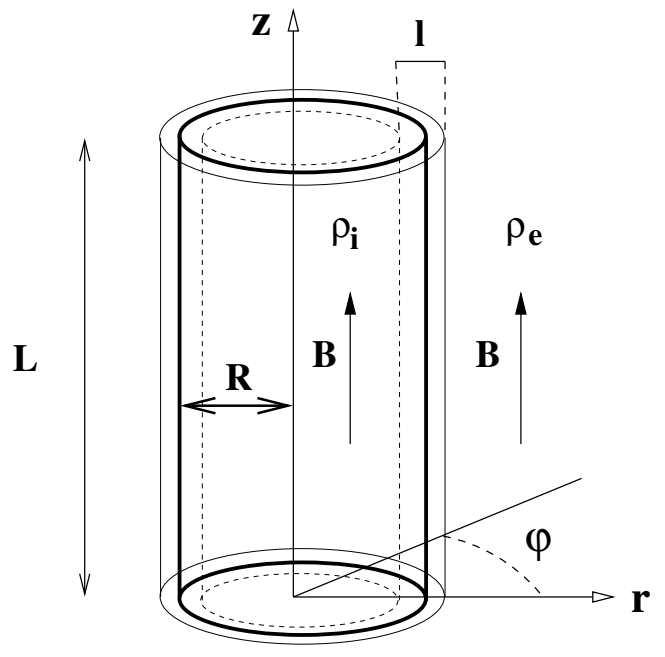

Fig. 1. Sketch of the equilibrium configuration representing a straightened coronal flux tube of length $L$ and radius $R$ modelled as a density enhancement. The magnetic field is uniform and parallel to the $z$-axis and the whole configuration is invariant in the $\varphi$-direction. The density varies in a non-uniform transitional layer of width $l$ from a constant internal value, $\rho_{\mathrm{i}}$ to a constant external value in the coronal environment, $\rho_{\mathrm{e}}$.

is that, in the thin boundary limit, the period and damping time are largely unaffected by the details of the longitudinal stratification of the density itself, but only depend on the weighted mean density.

In this paper, we study the resonant damping of coronal loop oscillations in fully two-dimensional configurations, with non-uniformity of the equilibrium density in both the radial and axial directions. The equilibrium model is fully non-uniform in the radial direction and the transitional layer can cover the whole loop. The linear resistive MHD wave equations are solved and the frequency and damping rate of the fundamental fast kink quasi-mode have been computed for a wide range of several loop parameter values, such as the length of the tube, the longitudinal stratification parameter, the density contrast, and the length-scale of the inhomogeneity.

The paper is organised as follows. In Sect. 2, the equilibrium model for the coronal flux tube and the equations of linear resistive MHD are given. The numerical method used for solving the linear resistive MHD equations is described in Sect. 3. The results of our numerical computations are given in Sect. 4. An overview of the dependence of the period and its damping rate on the different loop parameters is presented in Sects. 4.1-4.3. Section 4.4 focuses on the particular situation for highly non-uniform loops. Finally, in Sect. 5, our conclusions are drawn.

\section{Equilibrium and linear resistive MHD waves}

A coronal loop is modelled as a gravity-free, straight, cylindrically symmetric flux tube. In a system of cylindrical coordinates $(r, \varphi, z)$ with the $z$-axis coinciding with the axis of the cylinder (loop), the magnetic field is pointing in the $z$-direction (see Fig. 1). Since the plasma pressure is much smaller than the magnetic pressure in the corona, it is a good approximation 

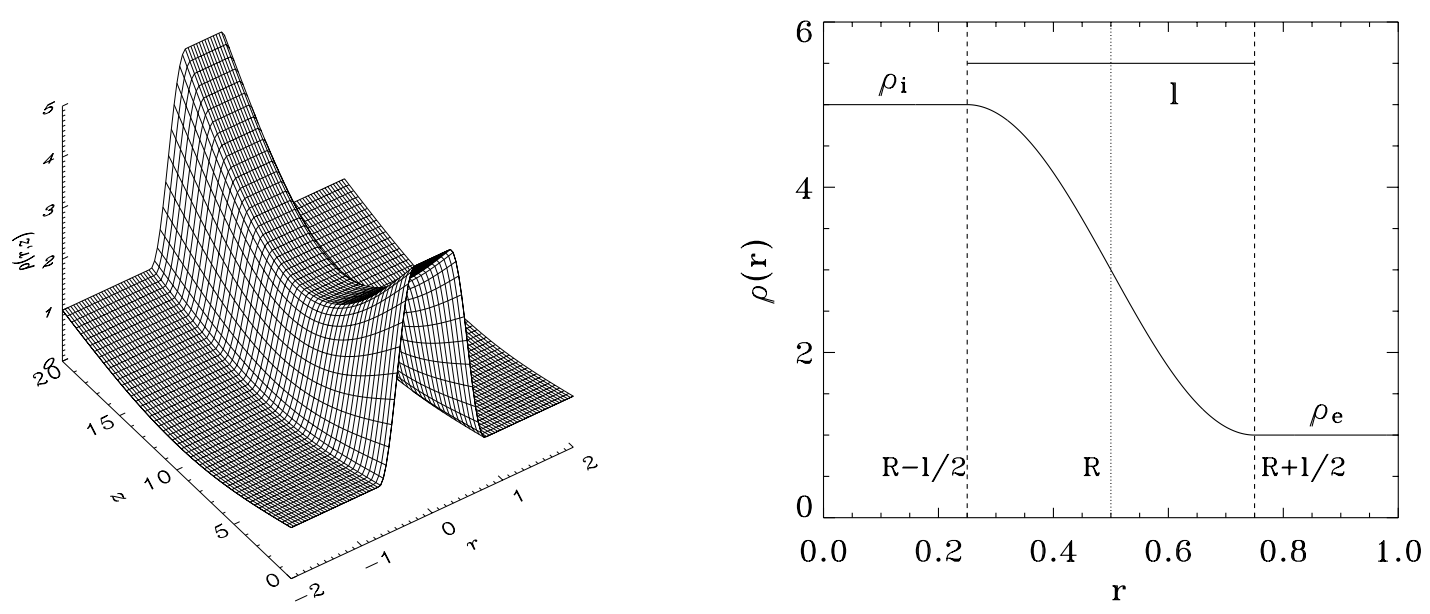

a

Fig. 2. a) Surface plot of the equilibrium density, $\rho(r, z)$, given by Eq. (1) in a coronal loop with $\zeta=5, l / R=1$ and $\alpha=0.5$. b) Radial dependence of the density at the footpoint $z=0$ showing the locations of the inner radius, $R-l / 2$, and the outer radius, $R+l / 2$.

to neglect plasma pressure. As the magnetic field is straight, this classic $\beta=0$ approximation implies that the magnetic field is uniform, $\boldsymbol{B}=\boldsymbol{B} \hat{\boldsymbol{e}}_{z}$, and also that the density, $\rho(r, z)$, or Alfvén speed, $v_{\mathrm{A}}(r, z)$, profiles can be chosen arbitrarily. Here, we are interested in a two-dimensional variation of plasma density both across and along the coronal loop of the form

$\rho(r, z)=\rho(r)\left[1-\alpha \sin \left(\frac{\pi z}{L}\right)\right]$,

with a separable dependence in the radial direction and in the axial direction along the tube. For the radial dependence of the density in Eq. (1), we follow Ruderman \& Roberts (2002); VD04, and assume a non-uniform sinusoidal density profile that connects the constant internal, $\rho_{\mathrm{i}}$, and the constant external, $\rho_{\mathrm{e}}$, values of the density along a transitional layer of length $l$ such that

$\rho(r)=\left\{\begin{array}{lc}\rho_{\mathrm{i}} & \text { for } 0 \leq r<R-\frac{l}{2}, \\ \frac{\rho_{\mathrm{i}}}{2}\left[\left(1+\frac{1}{\zeta}\right)-\left(1-\frac{1}{\zeta}\right) \sin \frac{\pi(r-R)}{l}\right] \\ \text { for } R-\frac{l}{2} \leq r \leq R+\frac{l}{2}, \\ \rho_{\mathrm{e}} & \text { for } r>R+\frac{l}{2},\end{array}\right\}$

where $\zeta=\rho_{\mathrm{i}} / \rho_{\mathrm{e}}$ is the density contrast. Note that $\zeta$ is the inverse of the density ratio, $\chi=\rho_{\mathrm{e}} / \rho_{\mathrm{i}}$, used by Ruderman $\&$ Roberts (2002). Here, the density contrast always takes values larger than 1 (for over dense coronal loops), and the density contrast really becomes larger when $\zeta$ increases. As for the density variation in the longitudinal direction, the parameter $\alpha$ in Eq. (1) controls the gradient of stratification along field lines. In the present analysis only the first term in the sine expansion of $\rho$ is considered, i.e., the density profile along the tube has only one extremum. The corresponding two-dimensional distribution of the equilibrium density, for a given value of the density contrast and stratification parameter, is shown in Fig. 2a. Figure $2 \mathrm{~b}$ shows the radial dependence of the density of the tube, for $l=R$.
The equilibrium configuration is perturbed in order to study the small amplitude oscillations of the system. The perturbed quantities are Fourier analysed, in time and in the ignorable angular variable $\varphi$, by assuming a dependence of the form $\exp (i(m \varphi-\omega t))$. The linear resistive MHD equations together with the appropriate boundary conditions form an eigenvalue problem. Here $m$ (an integer) is the azimuthal wave number and $\omega$ the frequency. The modes with $m=1$ are called fast kink-modes. They are the only modes that displace the axis of the tube. As was pointed out by Goossens et al. (2002), in the presence of a non-uniform boundary layer, kink mode oscillations have always their frequency in the Alfvén continuum and, hence, are damped quasi-modes. The real part of the frequency is related to the oscillation period, $P=2 \pi / \omega_{\mathrm{R}}$, whilst the damping time is related to the negative imaginary part of the frequency, $\tau_{\mathrm{d}}=-1 / \omega_{\mathrm{I}}$.

\section{Numerical method}

The linear resistive MHD wave equations are too complicated to allow fully analytic solutions for equilibrium configurations that mimic reality. Analytical solutions for non-uniform equilibrium models might exist for special choices of the equilibrium profiles. This fact reflects that the assumption of a "thin boundary" is essential for analytical development. The consideration of "thick" transitional layers yields strong damping rates, since the damping increases as the non-uniform layer becomes thicker, in such a way that $\omega_{\mathrm{I}} \simeq \omega_{\mathrm{R}}$. Instead of using a Taylor expansion, similar to the one performed for previous one-dimensional equilibrium models (Hollweg \& Yang 1988; Tirry \& Goossens 1996), a numerical root finder could be used to obtain the solutions. The tricky part is that the jump relations, used in those previous studies, are derived for weak damping and cannot be generalised easily to strong damping, hence the eigenvalue problem has to be solved numerically. For this purpose, we use a numerical code (POLLUX) to solve the linear resistive MHD equations.

In POLLUX, finite elements are used for the discretisation in the radial direction. In the longitudinal direction, 


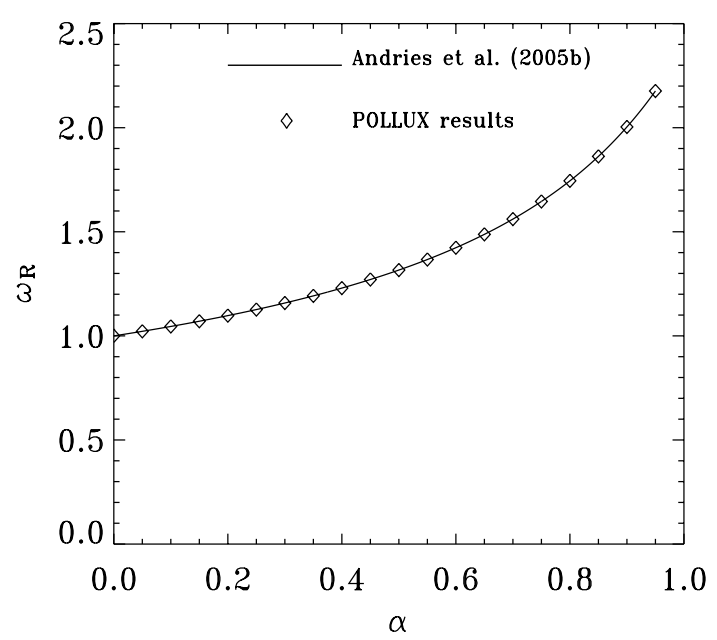

Fig. 3. Oscillation frequency, $\omega_{R}$, as a function of the stratification parameter, $\alpha$, in a coronal loop with $\zeta=2.0, L / R=157$, and $l / R=0.05$. Diamonds represent numerical solutions obtained with the POLLUX code. The continuous line is the solution obtained by Andries et al. (2005b). Note that the computed values do not reach $\alpha=1$ since this would correspond to zero density at the loop top. This unphysical value is not hence computed in this and subsequent calculations.

the perturbed quantities are represented by a finite number of Fourier modes of the form

$f(r, z)=\sum_{n=g-M}^{g+M} f_{n}(r) \mathrm{e}^{\imath \pi n z / L}$,

around the central mode $g$. Here $n$, an integer, is the longitudinal mode number. Although in POLLUX the equilibrium density is given analytically as a function of the $r$ - and $z$ - coordinates, by means of Eq. (1), and due to this longitudinal Fourier composition, the density profile needs to be Fourier-analysed in the $z$-direction in order to determine the influence on each Fourier mode in this direction. Therefore, there will be a small difference between the analytical profile and the numerical profile which is actually used in the code. In all our computations a number $M=15$ of modes has been found to be sufficient for the representation of the equilibrium density and the resulting spatial structure of the solutions in the longitudinal, $z$-direction.

Magnetic diffusivity, $\eta$, turns out to be an important parameter. In the numerical computation of resonantly damped quasi-modes, the correspondence of the ideal quasi-mode and the dissipative eigenmode is used, i.e. the fact that for sufficiently small resistivity, the damping becomes independent of resistivity, as shown by Poedts \& Kerner (1991). Hence, resistivity has to be small enough to assure that the damping rate is independent of dissipation, but large enough that the number of grid-points suffices to resolve the resonant layer. A parametric study of the damping rate as a function of resistivity was performed by VD04 for 1D non-uniform loop models. Figure 3 in VD04 shows that it is possible to find a range of values of resistivity such that the damping rate is independent of resistivity. For very small resistivity the convergence breaks down due to numerical errors, since there are not enough grid points to resolve the resonant layer. In all our computations the condition of independence on $\eta$ has to be fulfilled and values of resistivity in between $-\log \eta=6.0-7.0$ have been used. Dimensionless variables are used by normalising all lengths to the cylinder radius, $R$, whereas the density and the magnetic field are normalised to their respective equilibrium values on the cylinder axis. Herewith, speeds are normalised to the Alfvén speed on axis.

This paper focuses on fully non-uniform loops. Semianalytic investigation for longitudinal variation of density is possible when the variation across the loop is limited to a thin layer. This has been done by Andries et al. (2005b). An important result of that study is that the period and damping depend on an average mean density and are to a large extent insensitive to the details of the longitudinal stratification. An important purpose of this work is to check whether this theoretical prediction is confirmed for fully two-dimensional equilibrium models. In a first check we compare our results with those obtained by Andries et al. (2005b). Figure 3 displays the real part of the frequency as a function of the stratification parameter, $\alpha$, when the density at the footpoints is kept constant. In that case, both the real and imaginary parts of the frequency increase with $\alpha$. In order to perform such a comparison, the resulting frequencies have been normalised to the same value at $\alpha=0$ and there is a perfect agreement between the results of Andries et al. (2005b) and our computations with the POLLUX code.

\section{Numerical results}

Let us recall that our aim is to compute the frequency and damping rate of fast kink quasi-modes, for equilibrium models with longitudinal variation of the density and a thick nonuniform transitional layer in the radial direction.

We have obtained numerical solutions for wide ranges of the relevant parameters of the coronal loops. We try to cover the physical and geometrical properties of most of the coronal loops observed by Aschwanden et al. (2002). The relevant parameters and the ranges of variation selected in our computations are: the thickness of the inhomogeneous layer $(l / R \in[0.0-2.0])$, the inverse aspect ratio of the tube $(\varepsilon=$ $\pi R / L \in[0.02-0.18])$, the density contrast $(\zeta \in[1.5-5.0])$ and the longitudinal stratification parameter $(\alpha \in[0.0-1.0))$. The sampling of these four quantities is dictated by whether or not the period and damping rate are weakly or strongly sensitive to the variation of that particular quantity. The dependence of the period, damping time and damping rate of coronal loop oscillations on the inhomogeneity length-scale, the density contrast and the length of the tube, has already been studied in VD04. The only new parameter is the longitudinal stratification parameter. According to their results, a rather big spacing has been selected in the sampling of the data as a function of the length of the tube $(\varepsilon)$, the density contrast $(\zeta)$, and the stratification parameter $(\alpha)$. On the other hand, and due to the strong sensitivity of the period and damping time on the inhomogeneity length-scale, the spacing in $l / R$ has been chosen to be rather finer, $\Delta(l / R)=0.05$.

As for the stratification of the density in the axial direction, Andries et al. (2005b) have shown that the period and damping time are largely unaffected by the details of the longitudinal stratification of the density itself, but only depend on the 
weighted mean density. Hence, such as was done by Andries et al. (2005b) we have computed our eigenmodes by keeping the weighted mean density (weighted with the wave energy, $\left.\sin ^{2}(\pi z / L)\right)$ constant.

In the following first three subsections an overview of the dependence of the period and damping time as a function of these loop parameters is described. Then, in Sect. 4.4, coronal loops with near fully non-uniform radial density profiles are studied.

\subsection{Stratification and length of the loop}

In order to study the dependence of the period and damping time on the different loop parameters, we start with equilibrium models with fixed inhomogeneity length-scale, $l / R$, and fixed density contrast, $\zeta$, and look for the dependence on the stratification parameter, $\alpha$, and on the inverse aspect ratio of the loop, $\varepsilon$ (directly related to the length of the loop $L$ ). To this end, we take $l / R=1$ and $\zeta=5$. These are loops with thick non-uniform layers, that equal the radius of the loop, and with a rather high density contrast. Figures $4 a$ and $b$ show that the period of the oscillation decreases for decreasing length of the loop (increasing values of $\varepsilon$ ). The real part of the frequency (not shown here) varies almost linearly with $\varepsilon$. This is in agreement with analytical results obtained in coronal loops modelled as a discontinuous density enhancement (see Edwin \& Roberts 1983 , for example). The damping time shows a similar variation and, hence, is not shown here. Figure $4 \mathrm{~b}$ displays the normalised damping rate (a dimensionless variable independent of the normalisation made in time and space and related to the observable damping per period) as a function of the stratification parameter and the inverse aspect ratio. As expected, this quantity is almost independent of the length of the loop, since, as already stated both the period and damping time (or alternatively, the real and imaginary parts of the frequency) have the same behaviour with respect to this parameter. These results confirm the properties of period and damping time as a function of the length of the loop found by VD04, for 1D equilibrium models.

Let us now turn to the effect of longitudinal density stratification. Figure 4a shows that the period is almost independent of the stratification parameter. This is certainly true for values of $\alpha$ up to 0.8 or 0.9 . The same behaviour has been found for the damping time, hence, no dependence of the damping rate on the stratification parameter is seen in Fig. 4b. These two results confirm previous findings by Andries et al. (2005b). The slight dependence of the period and damping time on $\alpha$ for values near $0.8,0.9$ perfectly agrees with previous results, such as corresponds to the equilibrium with constant weighted mean density (weighted with the wave energy) we have chosen. Had we kept the density constant at the footpoints, a behaviour similar to the one displayed in Fig. 3 would be found. On the other hand, the independence of the normalised damping rate on the stratification parameter is due to the fact that longitudinal stratification is the same inside and outside the loop (see Andries et al. 2005b).

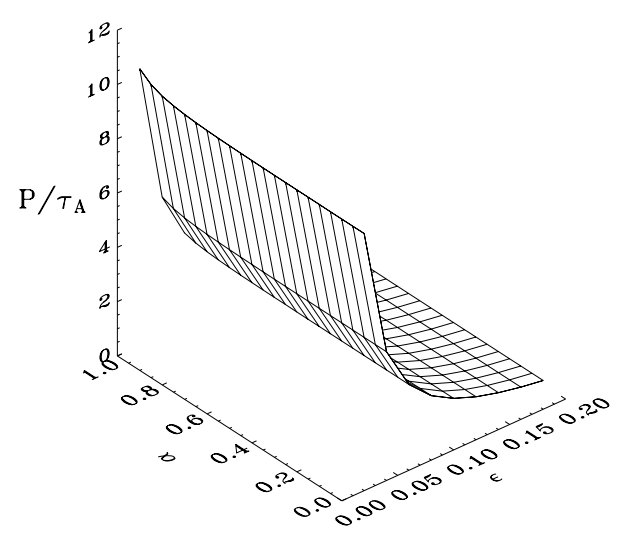

a

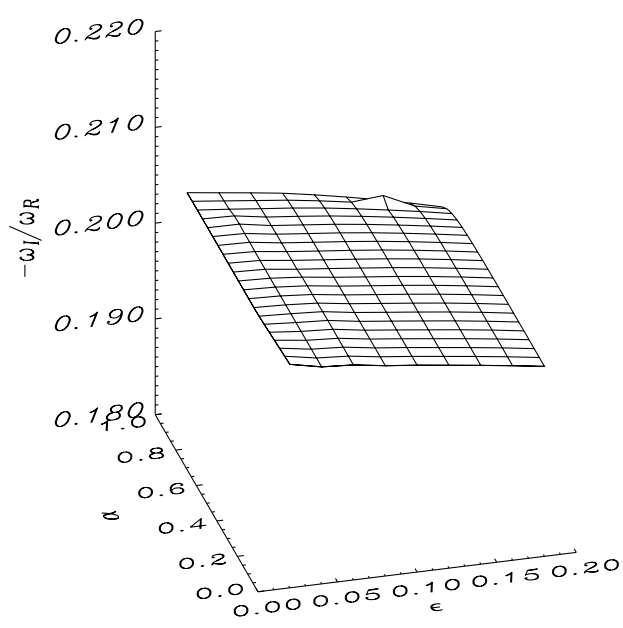

b

Fig. 4. a) Period and b) damping rate as a function of the inverse aspect ratio, $\varepsilon$, and longitudinal stratification parameter, $\alpha$, for coronal loops with fixed inhomogeneity length scale $(l / R=1.0)$ and fixed density contrast $(\zeta=5.0)$. In a) the normalisation made in space (normalising with respect to the radius $R$ ) and in speed (normalising with respect to the internal Alfvén speed $v_{\mathrm{A}}$ ) has been taken into account and, hence, the period is normalised to the Alfvén crossing time $\tau_{\mathrm{A}}$.

\subsection{Stratification and inhomogeneity length-scale}

Next, we look for the dependence of the period and damping time on the thickness of the inhomogeneous layer, $l / R$, and on the stratification parameter, $\alpha$. Hence, we consider coronal loops with a fixed length (fixed $\varepsilon=0.02$ ) and fixed density contrast $(\zeta=4.0)$. Figures $5 \mathrm{a}$ and $\mathrm{b}$ show that both the period and the damping time display the same behaviour with respect to the stratification parameter discussed in the previous section. The period and damping time are almost unaffected by the stratification parameter, at least for values below $\alpha \simeq 0.8$. A comparison between Figs. 5a and b shows that the period is more sensitive to the stratification for larger values of $\alpha$. On the other hand, Fig. 5c shows that the normalised damping rate is completely independent of the longitudinal stratification. In a similar manner as VD04, we can also compare the normalised damping rate, $-\omega_{\mathrm{I}} / \omega_{\mathrm{R}}$, with the value to be expected when the "thin tube and thin boundary" (TTTB) approximation is used. 


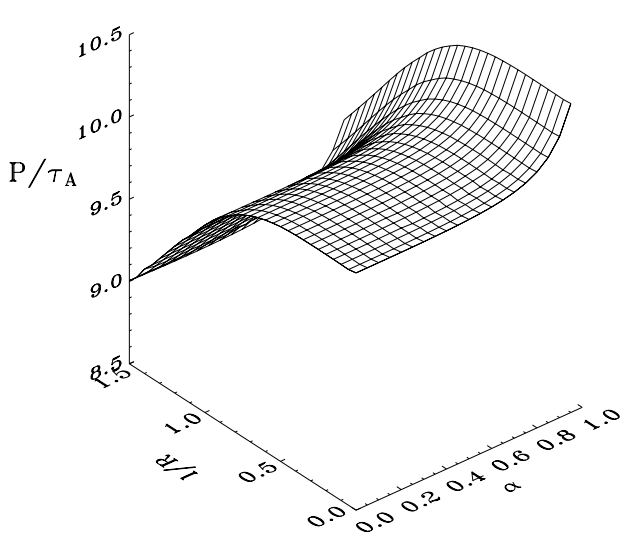

a

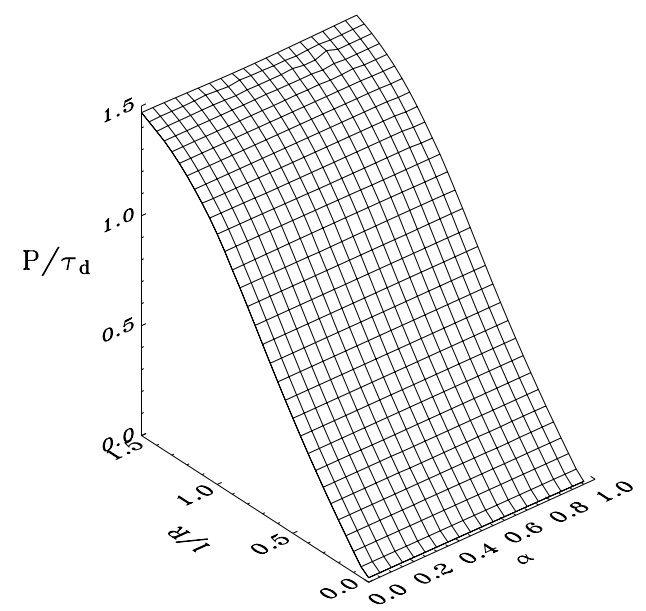

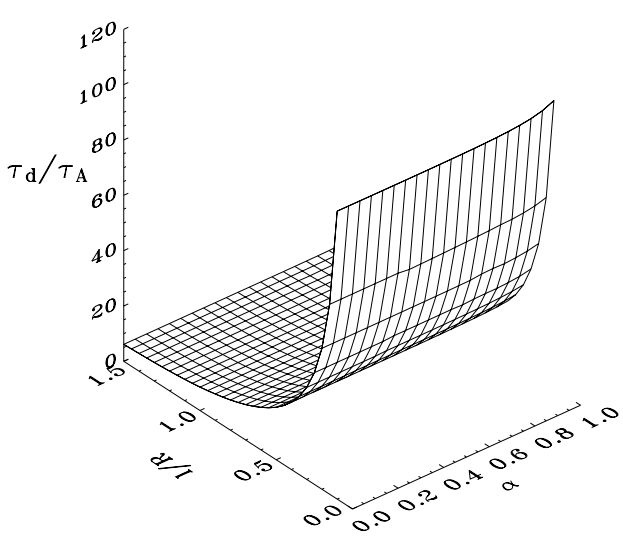

b

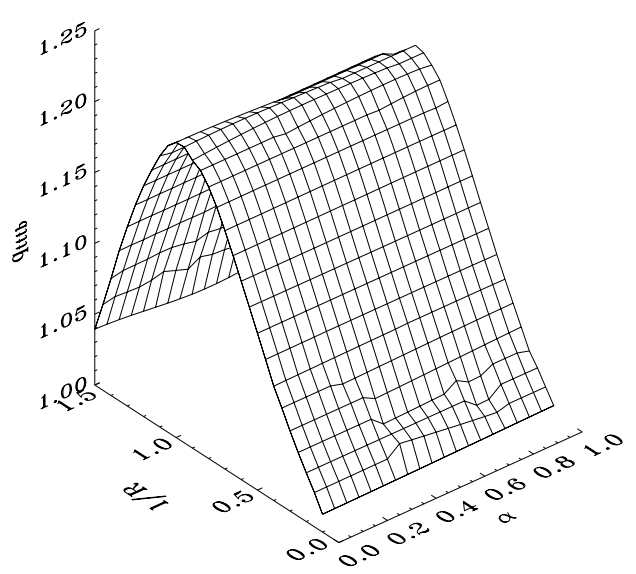

d

Fig. 5. a) Period, b) damping time, c) damping rate, and d) $q_{\text {tttb }}$ factor as a function of the stratification parameter and the thickness of the inhomogeneous boundary layer for coronal loops with fixed length $(\varepsilon=0.02)$ and density contrast $(\zeta=4.0)$. In a) and b) times are normalised to the Alfvén crossing time $\tau_{\mathrm{A}}$.

This is defined in one-dimensional equilibrium states by means of the factor $q_{\mathrm{tttb}}$ as

$\left(\frac{\omega_{\mathrm{i}}}{\omega_{\mathrm{r}}}\right)=-q_{\mathrm{tttb}} \frac{1}{4}\left(\frac{l}{R}\right)\left(\frac{\zeta-1}{\zeta+1}\right)$.

Figure 5d shows the two-dimensional distribution of this quantity as a function of $\alpha$ and $l / R$. The quantity $q_{\mathrm{tttb}}$ is independent of the stratification parameter, as it should be.

Regarding the influence of the inhomogeneity scale-length on the period and damping of coronal loop oscillations, Figs. 5a and $b$ show that the length of the inhomogeneous layer does have an important effect on both the period and damping time. In particular, the damping of the oscillation is very sensitive to the thickness of the boundary layer. This effect can also be appreciated in Fig. 5c that shows a monotonic increase of the damping rate with the inhomogeneity scale-length. As pointed out by VD04, for fully non-uniform 1D equilibrium models, the linear dependence of the damping rate on $l / R$ does not imply linear dependence on $l / a$ nor on $l / b$ (with $a=R+l / 2$ the outer radius and $b=R-l / 2$ the inner radius). This is because linear dependence is only valid on the "thin boundary" regime, i.e. $l / R \ll 1$, where the three formulas converge.
Finally, Fig. 5d shows the normalised damping rate (normalised with respect to the TTTB values). As for 1D equilibrium models we here find for 2D models that "thin tube and thin boundary" theory underestimates the damping rate even for moderate values of the inhomogeneity length scale. For small $l / R$ the damping rate has a linear dependence on $l / R$ which agrees very well with the results obtained with the analytical formula (Goossens et al. 2002) under the TTTB approximation. When $l / R$ is about 0.5 numerical results start to deviate significantly (over 5\%) (Van Doorsselaere et al. 2004). For intermediate $l / R$ (around 1) a maximum difference of almost $25 \%$ was found by VD04. For larger values of $l / R$ (see the decrease in Fig. 5d), TTTB overestimates the damping rate calculated with our numerical code.

\subsection{Stratification and density contrast}

Finally we report on the combined effects of the density contrast, $\zeta$, and of longitudinal stratification, $\alpha$. Hence we look at coronal loops with fixed length (fixed $\varepsilon=0.04$ ) and fixed thickness of the inhomogeneous layer $(l / R=1)$. The period and damping time as a function of $\alpha$ and $\zeta$ are shown in Figs. 6a 


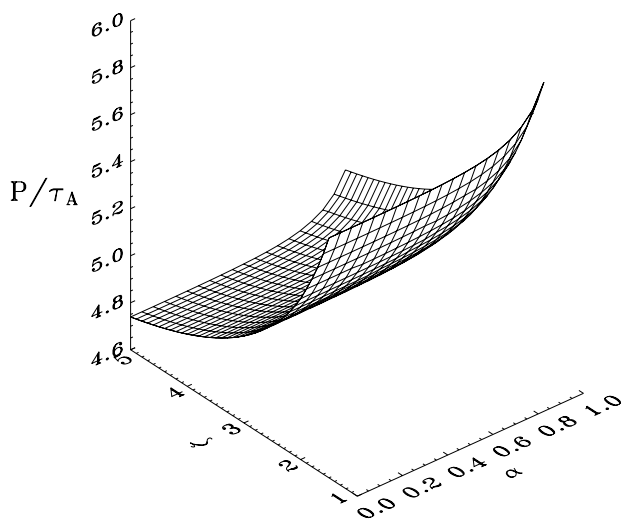

a

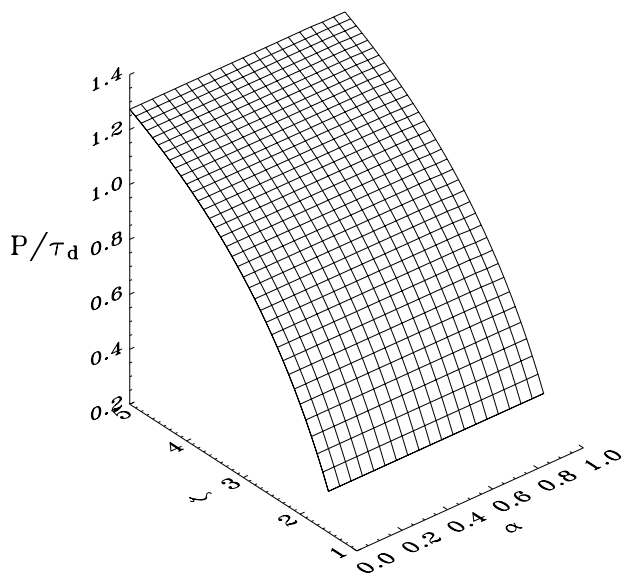

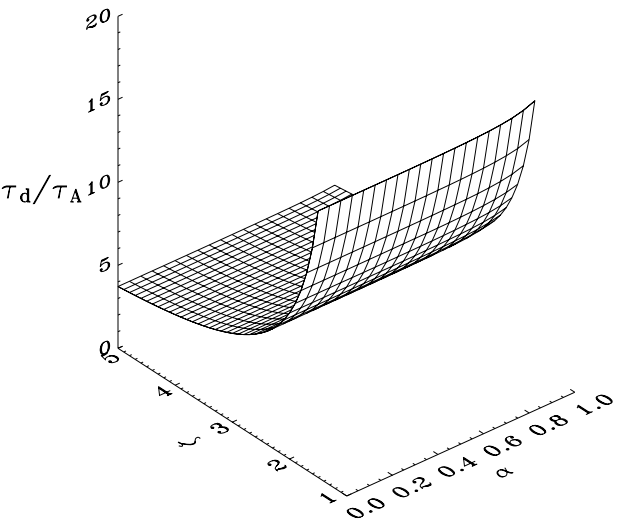

b

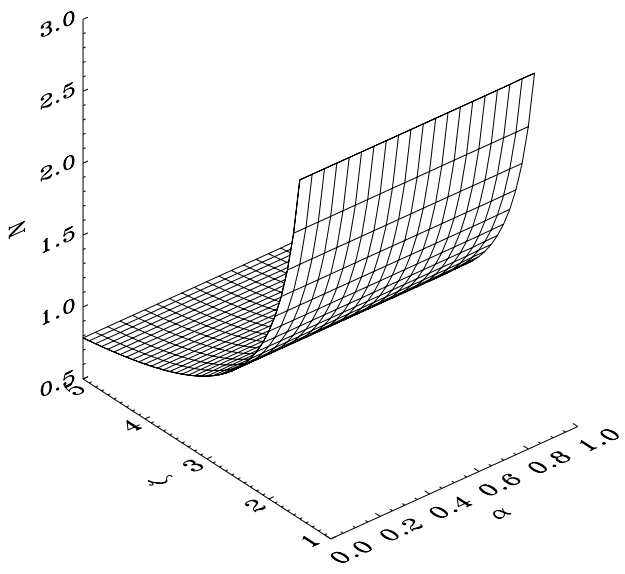

c

d

Fig. 6. a) Period, b) damping time, c) damping per period and d) number of oscillation periods, $N=\tau_{\mathrm{d}} / P$, as a function of the stratification parameter and the density contrast for coronal loops with a fixed length $(\varepsilon=0.04)$ and fixed thickness of the boundary layer $(l / R=1)$. In a) and $\mathbf{b})$ times are normalised to the Alfvén crossing time $\tau_{\mathrm{A}}$.

and $\mathrm{b}$. Concerning the density stratification, the behaviour obtained and described in the above two subsections is fully confirmed in the sense that the period and damping time are independent of $\alpha$ up to values near one. The normalised damping rate, shown in Fig. $6 \mathrm{c}$ shows no variation with longitudinal stratification in the full range of variation of $\alpha$.

On the other hand, Figs. $6 \mathrm{a}$ and $\mathrm{b}$ also show that the density contrast between the internal and the ambient plasma does affect the period and damping time. This strong dependency of period and damping rate was already found for 1D models by VD04. It shows up more clearly in the normalised damping rate (see Fig. 6c). The number of oscillations is given by $N=\tau_{\mathrm{d}} / P$ and is shown in Fig. 6d. This figure shows that $N$ is independent of stratification, but strongly depends on the loop density contrast. In particular, for the range of variation of the density contrast considered in our computations, coronal loop oscillations are very rapidly damped in a range of oscillatory periods from 0.78 to 2.85 . This result was predicted by Hollweg \& Yang (1988) and is in agreement with Aschwanden et al. (2003); VD04 who found that according to the strong dependence of the damping rate on the density contrast, in vacuum, the loop oscillations would be damped within a half oscillation period. Hence, the higher the ambient plasma density, the less severe damping by resonant absorption, so that undamped oscillations can only be supported if the density contrast is very small.

The numerical results discussed in the precedent three subsections show a behaviour of the period and damping of coronal loop oscillations as a function of the length of the tube, the thickness of the non-uniform boundary layer, and the density contrast that is in complete agreement with previous results obtained by VD04 for one-dimensional equilibrium models. The dependence with respect to longitudinal stratification turns out to confirm previous results obtained by Andries et al. (2005b) under the thin tube thin boundary approximation.

\subsection{Fully non-uniform coronal loops}

In the previous subsections, we have reported on results for models outside the "thin boundary" $(l / R \ll 1)$ regime. Up until now the largest value of $l / R$ is 1.5 . In this subsection, we concentrate on highly non-uniform loops and take values up to $l / R=2$.

Figure 7 a displays the normalised damping rate as a function of the density contrast, $\zeta$, and the inhomogeneity lengthscale, $l / R$, in coronal loops with $\varepsilon=0.14$ and longitudinal 


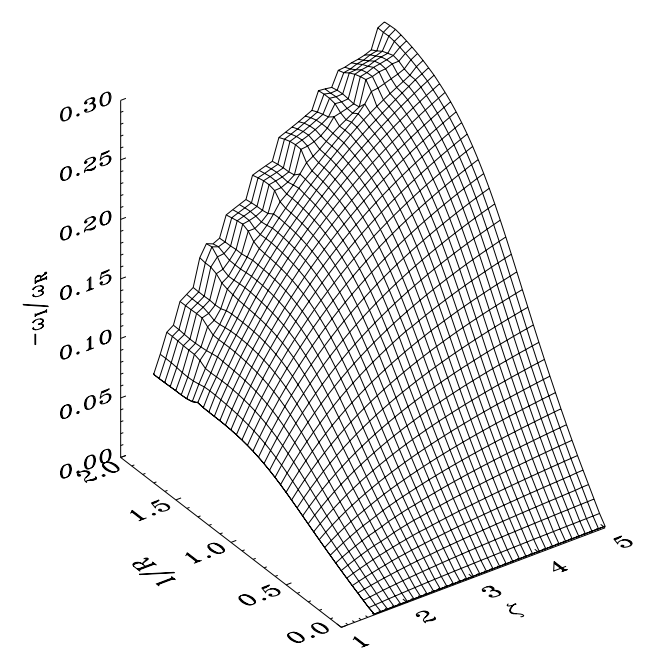

a

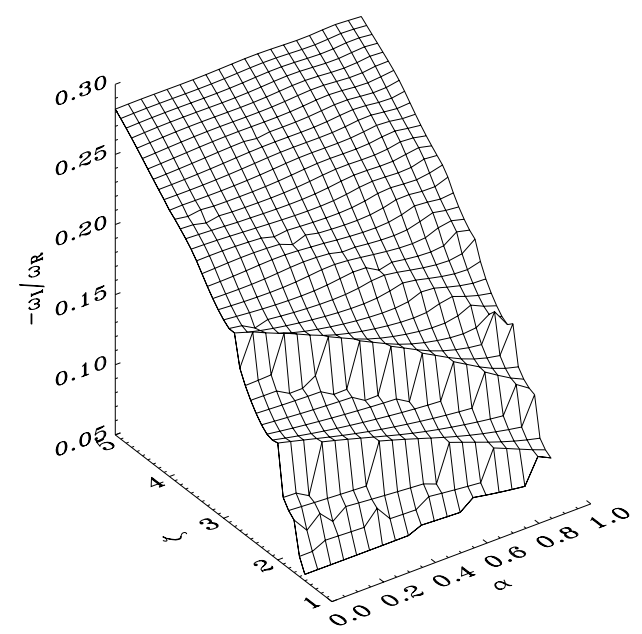

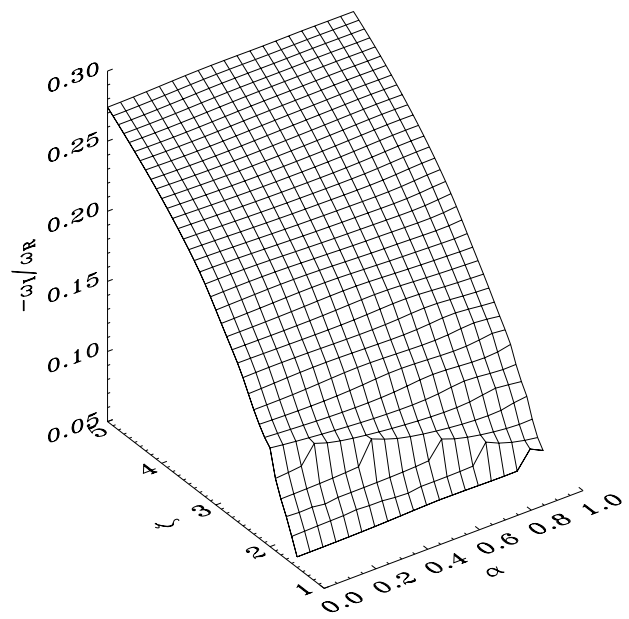

b

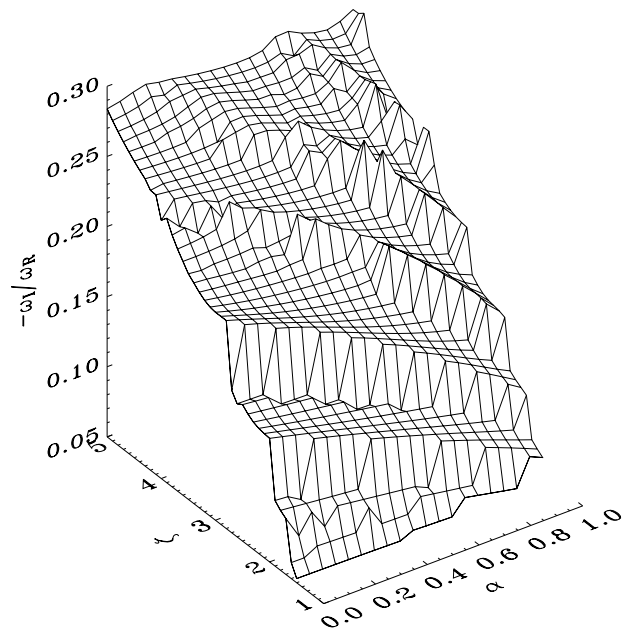

d

Fig. 7. a) Surface plot of the normalised damping rate as a function of the density contrast, $\zeta$ and inhomogeneity scale-length, $l / R$ for coronal loops with $\varepsilon=0.02$ and $\alpha=0.65$. b)-d) Surface plot of the normalised damping rate as a function of the stratification parameter, $\alpha$, and the density contrast, $\zeta$ for coronal loop equilibria with $\varepsilon=0.02$ and b) $l / R=1.65$, c) $l / R=1.85$ and d) $l / R=2.0$ (fully non-uniform loop). It can be appreciated that as soon as the inhomogeneity scale-length is larger than the previously considered values some wiggles appear and their presence seem to move until they fill the whole $(\alpha, \zeta)$ parameter plane.

stratification of the density, $\alpha=0.65$. As before, both the density contrast and the thickness of the transitional inhomogeneous layer have an important effect on the damping rate. Although the solution is smooth in most of the domain, some wiggles are visible in the region in which $l / R$ exceeds 1.65 . These wiggles are due to small gaps or jumps in the solution in the $(\zeta, l / R)$ plane for values of $l / R$ larger that 1.65 . These wiggles, for large values of $l / R$, have also been observed in other occasions. The clear relation between large values of the length scale of the inhomogeneity and the presence of those wiggles can be seen in Figs. 7b-d. On these figures we have plotted the normalised damping rate as a function of the stratification parameter, $\alpha$ and the density contrast, $\zeta$ for three different values of the inhomogeneity scale length in highly non-uniform loops, up to the full non-uniform case $(l / R=2)$. Wiggles seem to appear as soon as $l / R \simeq 1.65$ and, then, increase in number until the full domain of the computed solutions is filled up.
In order to understand these wiggles or gaps, we have determined the evolutionary tracks of the quasi-mode and of the resistive Alfvén modes in the complex frequency plane when $l / R$ increases. The result of this exercise is depicted in Fig. 8. Because of the change in the equilibrium model, the outer resistive Alfvén modes move inward, whereas the quasi-mode moves initially to the left. However, the quasi-mode avoids bumping into a resistive Alfvén mode and a deflection on a path parallel to that of the Alfvén modes is seen. At a given moment, for sufficiently large $l / R$, there is no obvious difference between the original quasi-mode and the resistive Alfvén modes.

A similar behaviour with interactions between quasi-modes and resistive Alfvén modes has been found in a somewhat different equilibrium model consisting of a Cartesian one-dimensional slab with a thick transitional layer by Van Doorsselaere et al. (2005). They have interpreted the 


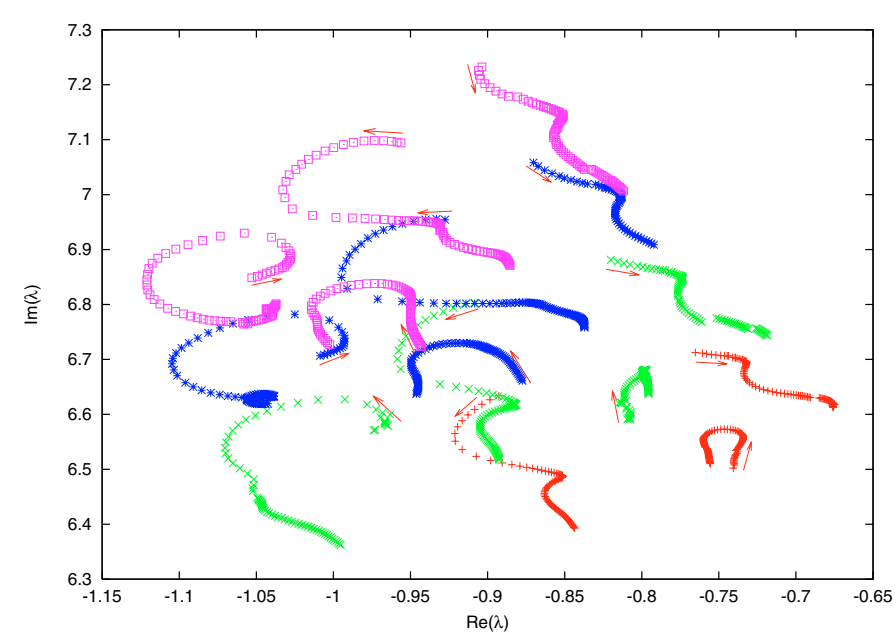

Fig. 8. The resistive spectrum in the complex frequency plane formed by the evolutionary tracks of the quasi-mode and the resistive Alfvén eigenmodes as the inhomogeneity scale-length increases from $l / R=$ 1.6 to $l / R=2$, in coronal loops with $\varepsilon=0.14$ and $\alpha=0.65$ (as in Fig. 7a), for four different values of the density contrast. $+: \zeta=2.0$, $x: \zeta=2.1, *: \zeta=2.2$, and boxes: $\zeta=2.3$. Arrows indicate the starting point of the frequencies and their initial direction as $l / R$ increases. It can be appreciated that the interaction between the quasi-mode is produced with the right-most Alfvén mode as the density contrast increases.

behaviour of the modes in terms of avoided crossings, since the modes seem to be forced to choose a side of the Alfvén mode either left or right on each of these encounters. Then, different gaps belong to interactions with different resistive Alfvén eigenmodes.

\section{Summary and conclusions}

In this paper, numerical solutions for resonantly damped MHD fast kink quasi-mode oscillations have been computed in twodimensional models of solar magnetic coronal loops. To this end, the classical cylindrical flux tube model has been considered with a two-dimensional equilibrium in which the density is allowed to vary both in the radial and longitudinal directions. Also, the assumption of a thin boundary layer that connects the external and internal parts of the tube has been removed. By solving the linearised resistive MHD wave equations a parametric numerical study of the fast kink-mode frequency and damping rate has been performed for a wide range of values for several loop parameters.

Numerical solutions have been computed for the period and damping of coronal loop oscillations for a wide range of values of several loop parameters, such as the density contrast, the length of the tube, the inhomogeneity scale length and the stratification coefficient. As has already been stated, this study constitutes an extension to the one performed by VD04 as it additionally considers longitudinal stratification of the equilibrium density. It also constitutes an extension to the computations done by Andries et al. (2005b) in the sense that the thickness of the non-uniform boundary layer is allowed to reach values up to $l / R=2$, hence we are not restricted to the thin boundary approximation.
The results from our computations in fully resistive twodimensional MHD have shown that the period and damping time of fast kink quasi-modes are almost independent of the longitudinal stratification parameter. The independence in $\alpha$ is maintained up to $\alpha \simeq 0.8$. On the other hand, the normalised damping rate, which is directly related to the observable damping per period is completely independent of these two parameters, $\varepsilon$ and $\alpha$. Indeed, the normalised damping rate remains unchanged, at least when stratification is the same inside and outside the coronal flux tube $(\alpha \neq \alpha(r))$, in perfect agreement with Andries et al. (2005b). Concerning the dependence on the inhomogeneity scale-length, we have confirmed the strong dependence of the damping on the thickness of the non-uniform boundary layer and, also, that the classical "thin tube and thin boundary" approximation underestimates the damping rate, by up to $20 \%$, for moderate values of $l / R$ (around 1). Finally, we have seen that the density contrast is a very important parameter, in such a way that large contrast loops get damped in less that an oscillatory period. Therefore, the results described in this paper represent a confirmation of previous findings obtained by VD04 in one-dimensional equilibrium states. Also, they constitute a confirmation of the analytical and semianalytical results obtained by Andries et al. (2005b) in their eigenmode computations for longitudinally stratified loops under the thin boundary approximation.

On the other hand, numerical solutions have been computed for highly non-uniform equilibria in which the inhomogeneity scale-length is larger than $l / R=1.5$ and can reach values up to the fully non-uniform case, $l / R=2$. It has been shown that in this case, the behaviour of the damping rate is quite different in the sense that gaps appear in the two-dimensional distribution of the normalised damping rate. By plotting the damping rate as a function of other parameters, such as the stratification parameter and the density contrast, while increasing the value of the inhomogeneity scale-length, the appearance of some wiggles in the solutions has been reported. The behaviour of these solutions is explained in terms of avoided crossings produced when changing the equilibrium parameters between the quasimode and the resistive Alfvén modes. These avoided crossings have also been found by Van Doorsselaere et al. (2005).

The results presented in this paper constitute an improvement on the theoretical understanding of quasi-mode damping over previous results obtained for one-dimensional loop models, since both stratification of the density along the loop and fully non-uniform radial profiles are considered. Concerning the possible application of the present results for an observational test similar to the one performed by Aschwanden et al. (2003), for 1D equilibrium models, our results do not give us additional information since, the assumed density stratification being radially independent, the normalised damping is independent of longitudinal density stratification. However, the present results can be of importance for coronal seismology in their application to observations of multi mode loop oscillations, such as the ones described by Verwichte et al. (2004). For instance, Andries et al. (2005a) have shown that longitudinal stratification has a different effect on the fundamental as on the first overtone, in such a way that in a stratified atmosphere the oscillation period of the fundamental mode should be less 
than double that of the first overtone mode. This fact has been used as a seismological tool to determine the longitudinal stratification in coronal loops. In that paper, only the observed and computed oscillatory periods were used. The results presented in this paper could be applied for a similar study using both the period and damping time.

Acknowledgements. I. Arregui acknowledges the financial support received in the framework of the project GOA/2004/01 (K.U. Leuven).

\section{References}

Andries, J., Arregui, I., \& Goossens, M. 2005a, ApJ, 624, L57

Andries, J., Goossens, M., Hollweg, J. V., Arregui, I., \& Van Doorsselaere, T. 2005b, A\&A, 430, 1109

Aschwanden, M. J., Fletcher, L., Schrijver, C. J., \& Alexander, D. 1999, ApJ, 520, 880

Aschwanden, M. J., De Pontieu, B., Schrijver, C. J., \& Title, A. M. 2002, Sol. Phys., 206, 99

Aschwanden, M. J., Nightingale, R. W., Andries, J., Goossens, M., \& Van Doorsselaere, T. 2003, ApJ, 598, 1375

Davila, J. M. 1987, ApJ, 317, 514

De Pontieu, B., Martens, P. C. H., \& Hudson, H. S. 2001, ApJ, 558, 859

Edwin, P. M., \& Roberts, B. 1983, Sol. Phys., 88, 179

Goossens, M., Andries, J., \& Aschwanden, M. J. 2002, A\&A, 394, L39

Grossmann, W., \& Smith, R. A. 1988, ApJ, 332, 476
Heyvaerts, J., \& Priest, E. R. 1983, A\&A, 117, 220

Hollweg, J. V., \& Yang, G. 1988, J. Geophys. Res., 93, 5423

Ionson, J. A. 1978, ApJ, 226, 650

Lee, M. A., \& Roberts, B. 1986, ApJ, 301, 430

Nakariakov, V. M., \& Ofman, L. 2001, A\&A, 372, L53

Nakariakov, V. M., Ofman, L., DeLuca, E. E., Roberts, B., \& Davila, J. M. 1999, Science, 285, 862

Ofman, L. 2002, ApJ, 568, L135

Ofman, L., \& Aschwanden, M. J. 2002, ApJ, 576, L153

Ofman, L., Davila, J. M., \& Steinolfson, R. S. 1994, ApJ, 421, 360

Poedts, S., \& Kerner, W. 1991, Phys. Rev. Lett., 66, 2871

Priest, E. R. 1982, Solar Magnetohydrodynamics, Chapters 4-6 (Dordrecht: D. Reidel Publishing)

Roberts, B. 2000, Sol. Phys., 193, 139

Roberts, B., Edwin, P. M., \& Benz, A. O. 1984, ApJ, 279, 857

Ruderman, M. S., \& Roberts, B. 2002, ApJ, 577, 475

Schrijver, C. J., \& Brown, D. S. 2000, ApJ, 537, L69

Schrijver, C. J., Aschwanden, M. J., \& Title, A. M. 2002, Sol. Phys., 206, 69

Steinolfson, R. S., \& Davila, J. M. 1993, ApJ, 415, 354

Tirry, W. J., \& Goossens, M. 1996, ApJ, 471, 501

Uchida, Y. 1970, PASJ, 22, 341

Van Doorsselaere, T., Andries, J., Poedts, S., \& Goossens, M. 2004, ApJ, 606, 1223 (VD04)

Van Doorsselaere, T., Goossens, M., Andries, J., \& Poedts, S. 2005, in preparation

Verwichte, E., Nakariakov, V. M., Ofman, L., \& Deluca, E. E. 2004, Sol. Phys., 223, 77 